\title{
REAÇÃO DE BIS-INSERÇÃo DE 1,2-DIFENILACETILENO NA LIGAÇÃo Pd-C DE CICLOMETALADOS
}

Sandra Regina Ananias, Anderson Martinez Santana e Antonio Eduardo Mauro*

Departamento de Química Geral e Inorgânica, Instituto de Química, Universidade Estadual Paulista, CP 355, 14801-970

Araraquara - SP

Vicente Alexandre de Lucca Neto

Departamento de Ciências Exatas e Naturais, Universidade do Sagrado Coração, Bauru - SP

Eduardo Tonon de Almeida

Curso de Química, Universidade Católica de Brasília, CP 5490, 72030-170 Brasília - DF

Recebido em 1/3/02; aceito em 12/6/02

\begin{abstract}
BIS INSERTION REACTION OF 1,2-DIPHENYLACETILENE INTO Pd-C BOND OF CYCLOMETALLATED SPECIES. The present paper deals with the bis-insertion reactions of 1,2-diphenylacetylene into Pd-C bond of the cyclopalladated complexes $[\mathrm{Pd}(\mathrm{dmba})(\mu-\mathrm{NCO})]_{2}(\mathbf{1})$ and $\left[\mathrm{Pd}(\mathrm{dmba})(\mathrm{MeCN})_{2}\right]\left(\mathrm{NO}_{3}\right)(\mathbf{2})(\mathrm{dmba}=\mathrm{N}, \mathrm{N}$-dimethylbenzylamine, $\mathrm{MeCN}=$ acetonitrile $)$. Two new complexes $\left[\mathrm{Pd}\left\{\mathrm{PhC}=\mathrm{CPh}-\mathrm{CPh}=\mathrm{CPhC}_{6} \mathrm{H}_{4} \mathrm{CH}_{2} \mathrm{~N}\left(\mathrm{CH}_{3}\right)_{2}\right\}(\mathrm{NCO})\right](3)$ and $\left.\left[\mathrm{Pd}\left\{\mathrm{PhC}=\mathrm{CPh}-\mathrm{CPh}=\mathrm{CPhC}_{6} \mathrm{H}_{4} \mathrm{CH}_{2} \mathrm{~N}_{\left(\mathrm{CH}_{3}\right.}\right)_{2}\right\}\left(\mathrm{NO}_{3}\right)\right]$ (4) were obtained and characterized by IR and NMR spectroscopy and elemental analysis.
\end{abstract}

Keywords: 1,2-diphenylacetylene; cyclopalladated; bis-insertion.

\section{INTRODUÇÃO}

Os últimos anos evidenciam um crescente interesse na Química de compostos organometálicos, notadamente dos ciclometalados, pelas suas vastas e diversificadas aplicações como em processos catalíticos $^{1}$, materiais mesogênicos ${ }^{2}$, na área médica ${ }^{3}$ e mais recentemente na química supramolecular ${ }^{4}$. O termo ciclometalado, introduzido por Trofimenko ${ }^{5}$, descreve reações nas quais ligantes orgânicos sofrem uma reação de metalação intramolecular levando à formação de um anel quelato, com uma ligação de coordenação entre o metal $\mathrm{M}$ e um átomo doador $\mathrm{Y}$ dos grupos $\mathrm{V}(\mathrm{N}, \mathrm{P}, \mathrm{As})$ ou $\mathrm{VI}(\mathrm{O}, \mathrm{S}, \mathrm{Se})$, e uma ligação covalente metal-carbono, sendo $\mathrm{X}$ um halogênio ou grupo alquil, como ilustrado na Figura 1.

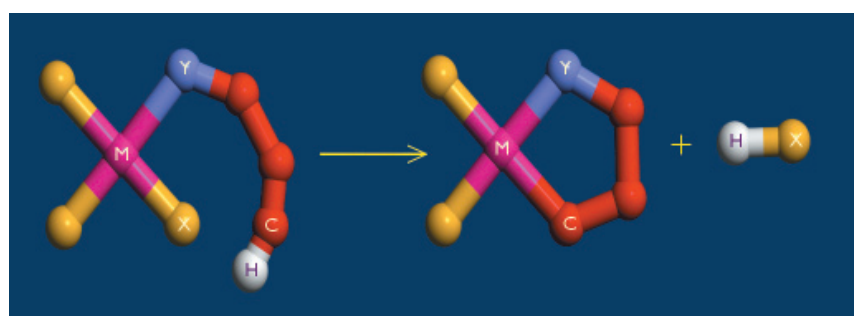

Figura 1. Representação esquemática da formação do anel ciclometalado

A labilidade da ligação M-C é de grande interesse em síntese orgânica e, nos últimos anos, várias reações de complexos ortometalados com alcenos, alcinos, monóxido de carbono, isocianetos e halogênios forneceram rotas importantes para a obtenção de uma variedade de moléculas aromáticas 1,2-dissubstituídas e heterociclos, entre outras ${ }^{6}$. Particularmente os ciclometalados diméricos, contendo haletos em ponte, são adequados para a síntese de novos compostos mediante reações com nucleófilos ${ }^{7}$, além de apresentarem inserções de alcenos $^{8}$ e alcinos $^{9}$ na ligação Pd-C.

*e-mail: mauro@iq.unesp.br
É bem conhecido que a inserção de alcinos nas ligações $\mathrm{Pd}-\mathrm{C}_{\mathrm{sp}^{3}}$ e Pd- $\mathrm{Csp}_{\mathrm{sp}^{2}}$ de compostos de coordenação intramolecular originam produtos contendo a incorporação de uma, duas ou três unidades de alcino na ligação $\mathrm{Pd}-\mathrm{C}^{10}$. Recentemente, realizamos vários estudos com ciclopaladados e, em dois deles, o ânion heterociclo $\mathrm{CN}_{3} \mathrm{~S}_{2}^{-}$resultou da interação de $\mathrm{CS}_{2}$ com o grupo azido coordenado ao paládio nos compostos $\left[\mathrm{Pd}(\mathrm{dmba})\left(\mu-\mathrm{N}_{3}\right)\right]_{2}{ }^{11}$ e $\left[\mathrm{Pd}(\mathrm{bzan})\left(\mu-\mathrm{N}_{3}\right)\right]_{2}{ }^{12}$ $(\mathrm{dmba}=\mathrm{N}, \mathrm{N}$-dimetilbenzilamina e bzan $=\mathrm{N}$-benzilidenoanilina $)$. A presente investigação relaciona-se com este contexto e trata da inserção de difenilacetileno (dfa) na ligação $\sigma \mathrm{Pd}-\mathrm{C}$ de $[\operatorname{Pd}(\mathrm{dmba})(\mu-$ $\mathrm{NCO})]_{2}^{13}(\mathbf{1})$ e $\left[\mathrm{Pd}(\mathrm{dmba})(\mathrm{MeCN})_{2}\right]\left(\mathrm{NO}_{3}\right)^{14}(\mathbf{2})$, o que propiciou a obtenção de um fragmento orgânico maior coordenado ao paládio.

\section{PARTE EXPERIMENTAL}

Todas as reações foram realizadas à temperatura ambiente, utilizando-se técnicas de Schlenk e atmosfera de $\mathrm{N}_{2}$, com os reagentes, todos de grau analítico, empregados sem purificação prévia. Os produtos, depois de isolados, são estáveis ao ar e à umidade.

\section{Preparação dos compostos}

Os compostos $[\mathrm{Pd}(\mathrm{dmba})(\mu-\mathrm{NCO})]_{2}(\mathbf{1})$ e $\left[\mathrm{Pd}(\mathrm{dmba})(\mathrm{MeCN})_{2}\right]$ $\left(\mathrm{NO}_{3}\right)(2)$ foram preparados como descrito na literatura ${ }^{13,14}$.

$$
\left[\mathrm{Pd}\left\{\mathrm{PhC}=\mathrm{CPh}-\mathrm{CPh}=\mathrm{CPhC}_{6} \mathrm{H}_{4} \mathrm{CH}_{2} \mathrm{~N}\left(\mathrm{CH}_{3}\right)_{2}\right\}(\mathrm{NCO})\right](\mathbf{3})
$$

Uma massa de $0,100 \mathrm{~g}(0,177 \mathrm{mmol})$ de $[\mathrm{Pd}(\mathrm{dmba})(\mu-\mathrm{NCO})]_{2} \mathrm{e}$ $50 \mathrm{~mL}$ de diclorometano foram adicionados em um Schlenk sob atmosfera de $\mathrm{N}_{2}$. Posteriormente adicionou-se à suspensão, sob agitação, 0,1292 g (0,725 mmol) de difenilacetileno. A solução permaneceu sob agitação por $9 \mathrm{~h}$, tornando-se amarela escura e límpida. A solução foi então concentrada em rota-vapor a $3 / 4$ de seu volume inicial, ocorrendo a precipitação de um composto amarelo ouro pela adição de pentano. O complexo foi filtrado, lavado com pentano e seco sob vácuo. Rendimento: $87 \%$. Análise elementar: \% calc. (\% enc.): C: 71,42 (70,88); H: 5,05 (5,04); N: 4,38 (4,10). 
$\left[\mathrm{Pd}\left\{\mathrm{PhC}=\mathrm{CPh}-\mathrm{CPh}=\mathrm{CPhC}_{6} \mathrm{H}_{4} \mathrm{CH}_{2} \mathrm{~N}\left(\mathrm{CH}_{3}\right)_{2}\right\}\left(\mathrm{NO}_{3}\right)\right] .1,5 \mathrm{CH}_{2} \mathrm{Cl}_{2}(4)$

Uma massa de $0,100 \mathrm{~g}(0,260 \mathrm{mmol})$ de $\left[\mathrm{Pd}(\mathrm{dmba})(\mathrm{MeCN})_{2}\right]$ $\left(\mathrm{NO}_{3}\right)$ e $50 \mathrm{~mL}$ de diclorometano foram adicionados em um Schlenk sob atmosfera de $\mathrm{N}_{2}$. Posteriormente adicionou-se à suspensão, sob agitação, 0,180 g (1,04 mmols) de difenilacetileno (dfa). A mistura reacional foi mantida sob agitação durante $9 \mathrm{~h}$ e então, evaporou-se o solvente até quase a secura observando-se a formação de um sólido amarelo. Este sólido foi posteriormente lavado com diclorometano e pentano e seco sob vácuo. Uma massa de $0,190 \mathrm{~g}$ do produto foi obtida, equivalente a aproximadamente $90 \%$ de rendimento, após a recristalização, utilizando diclorometano como solvente e pentano como agente precipitante. Análise Elementar: calc. \% (enc.): C: 67,4 $(68,5) ; \mathrm{H}: 4,90(5,22)$; N: 4,25 (4,05).

\section{Técnicas experimentais}

As análises quantitativas dos elementos carbono, hidrogênio e nitrogênio foram efetuadas no analisador automático EA 1110 CHNS$\mathrm{O}$ da CE-Instruments.

Os espectros de absorção na região do IV foram obtidos em um espectrofotômetro NICOLET FT-IR IMPACT 400, no intervalo de $4000-400 \mathrm{~cm}^{-1}$ e com resolução de $4 \mathrm{~cm}^{-1}$, em pastilhas de $\mathrm{KBr}$.

Os espectros de RMN de ${ }^{1} \mathrm{H}$ e ${ }^{13} \mathrm{C}$ foram obtidos em um espectrofotômetro BRUCKER AC-200, empregando-se as frequiências de $200 \mathrm{MHz}$ e $51 \mathrm{MHz}$, respectivamente. O solvente empregado foi o clorofórmio deuterado $\left(\mathrm{CDCl}_{3}\right)$ e, como padrão interno, o tetrametilsilano (TMS).

\section{RESULTADOS E DISCUSSÃO}

\section{Espectros no IV e de RMN}

Os ciclopaladados $[\mathrm{Pd}(\mathrm{dmba})(\mu-\mathrm{NCO})]_{2} \quad$ (1) e $\left[\mathrm{Pd}(\mathrm{dmba})(\mathrm{MeCN})_{2}\right]\left(\mathrm{NO}_{3}\right)(2)$ reagem, em diclorometano e sob atmosfera de $\mathrm{N}_{2}$, com difenilacetileno, em excesso, originando os produtos (3) e (4) ambos amarelos e com altos rendimentos, de acordo com as equações abaixo:

$[\mathrm{Pd}(\mathrm{dmba})(\mathrm{NCO})]_{2}+4 \mathrm{dfa} \rightarrow 2\left[\mathrm{Pd}\left\{\mathrm{PhC}=\mathrm{CPh}-\mathrm{CPh}=\mathrm{CPhC}_{6} \mathrm{H}_{4}\right.\right.$ $\left.\left.\mathrm{CH}_{2} \mathrm{~N}\left(\mathrm{CH}_{3}\right)_{2}\right\}(\mathrm{NCO})\right]$

$\left[\mathrm{Pd}(\mathrm{dmba})\left(\mathrm{CH}_{3} \mathrm{CN}\right)_{2}\right]\left(\mathrm{NO}_{3}\right)+2 \mathrm{dfa} \rightarrow\left[\mathrm{Pd}\left\{\mathrm{PhC}=\mathrm{CPh}-\mathrm{CPh}=\mathrm{CPhC}_{6} \mathrm{H}_{4}\right.\right.$ $\left.\left.\mathrm{CH}_{2} \mathrm{~N}\left(\mathrm{CH}_{3}\right)_{2}\right\}\left(\mathrm{NO}_{3}\right)\right]+2 \mathrm{CH}_{3} \mathrm{CN}$

Os resultados de análise elementar são consistentes com a adição de dois alquinos para cada centro metálico, sendo a regioespecificidade alcançada mediante o emprego de difenilacetileno (dfa) em excesso e longos períodos reacionais, o que impediu a formação de misturas contendo produtos de mono e bis-inserção.

Os compostos (3) e (4) foram devidamente investigados pelas espectroscopias de absorção no infravermelho e RMN de ${ }^{1} \mathrm{H} \mathrm{e}{ }^{13} \mathrm{C}$, estando os principais aspectos apresentados a seguir.
A espectroscopia no infravermelho mostrou-se extremamente útil para esclarecer os modos de coordenação dos íons $\mathrm{NCO}^{-}$e $\mathrm{NO}_{3}^{-}$ao paládio nos compostos (3) e (4), respectivamente. As frequiências vibracionais $\left(\mathrm{cm}^{-1}\right)$ mais significativas para inferir aspectos estruturais dos complexos são dadas na Tabela 1 .

A presença de uma banda intensa no espectro de (1), em $2175 \mathrm{~cm}^{-1}$, atribuída ao modo vibracional $v_{\text {as }}(\mathrm{NCO})$, indica claramente que o grupo $\mathrm{NCO}^{-}$encontra-se coordenado em ponte, através do átomo de nitrogênio, a dois centros metálicos ${ }^{13}$. Nota-se, contudo, que no espectro do composto (3) esta banda é deslocada para $2220 \mathrm{~cm}^{-1}$, um valor típico referente a $\mathrm{NCO}^{-}$terminal, $\mathrm{N}(2)-\mathrm{C}(8)-\mathrm{O}$, conforme mostrado na Figura 2, coordenado via átomo de nitrogênio N(2) ao centro metálico ${ }^{15}$. Este dado também indica que a espécie dinuclear (1), ao reagir com dfa, deu origem a uma outra mononuclear; fato consubstanciado adiante por dados de RMN de ${ }^{1} \mathrm{H}$ e ${ }^{13} \mathrm{C}$. No espectro IV de (3) não aparece nenhuma banda associada ao $v(\mathrm{C} \equiv \mathrm{C})$ do dfa. Observa-se, por outro lado, uma banda em $1595 \mathrm{~cm}^{-1}$, atribuída ao modo vibracional $v(\mathrm{C}=\mathrm{C})$; fato comprobatório da inserção do dfa [carbonos $\mathrm{C}(1)$ a $\mathrm{C}(4)$ ] na ligação $\sigma \mathrm{Pd}-\mathrm{C}(5)$ do composto (1).

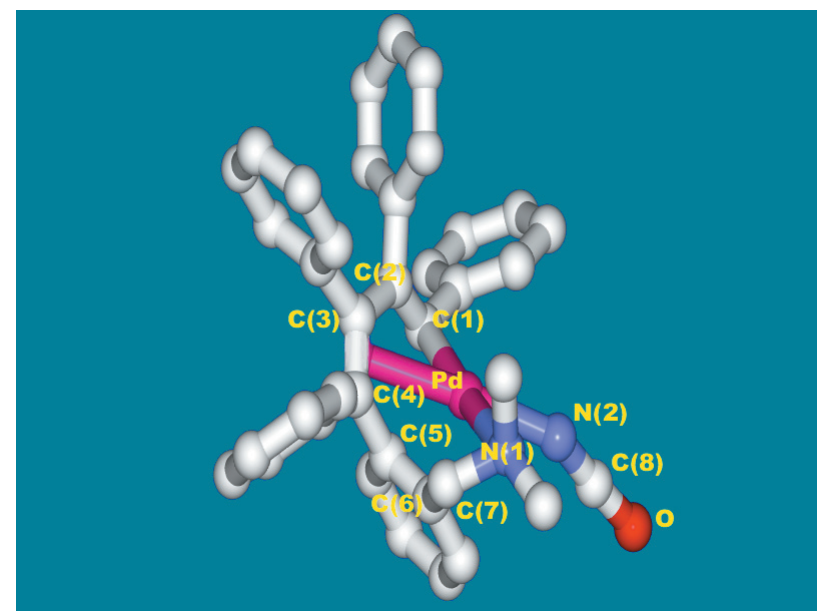

Figura 2. Produto de bis-inserção de difenilacetileno na ligação Pd-C no composto $[\mathrm{Pd}(\mathrm{dmba})(\boldsymbol{\mu}-\mathrm{NCO})]$

O espectro IV de (2) mostra bandas em 1387 e $860 \mathrm{~cm}^{-1}$, atribuídas aos modos $v(\mathrm{ONO})$ e $\delta(\mathrm{ONO})$, respectivamente, e indicativas de nitrato iônico ${ }^{14,15}$.

No espectro IV de (4) aparecem bandas associadas ao $\mathrm{NO}_{3}^{-} \mathrm{em}$ 1394, 1267 e $799 \mathrm{~cm}^{-1}$, as duas primeiras atribuídas a modos de estiramento $v(\mathrm{ONO})$ e a última assinalada à deformação $\delta(\mathrm{ONO})$. Estas três bandas evidenciam a coordenação do grupo $\mathrm{NO}_{3}^{-}$de forma monodentada ao paládio ${ }^{15}$. Nota-se também uma banda em $1590 \mathrm{~cm}^{-1}$ atribuída ao $v(\mathrm{C}=\mathrm{C})$, sugerindo a inserção do difenilacetileno na ligação $\sigma \mathrm{Pd}-\mathrm{C}(5)$ de (2). Nenhuma banda referente ao ligante $\mathrm{CH}_{3} \mathrm{CN}$, presente no complexo (2), foi notada no espectro de (4), comprovan-

Tabela 1. Frequiências vibracionais dos espectros no IV $\left(\mathrm{cm}^{-1}\right)$ para os compostos $\mathbf{1}$ - $\mathbf{4}$

\begin{tabular}{|c|c|c|c|c|c|}
\hline Composto & $v_{\mathrm{as}}(\mathrm{NCO})$ & $v_{s}(\mathrm{NCO})$ & $v(\mathrm{ONO})$ & $\delta(\mathrm{NCO})$ & $v(\mathrm{C}=\mathrm{C})$ \\
\hline$[\mathrm{Pd}(\mathrm{dmba})(\mu-\mathrm{NCO})]_{2}(\mathbf{1})$ & 2175 vs & $1287 \mathrm{w}$ & - & $683 \mathrm{w}, 596 \mathrm{w}$ & $\ldots$ \\
\hline$\left[\mathrm{Pd}(\mathrm{dmba})(\mathrm{MeCN})_{2}\right]\left[\mathrm{NO}_{3}\right](\mathbf{2})$ & & {[} & 1387 vs & $\ldots$ & \\
\hline$\left[\mathrm{Pd}\left\{\mathrm{PhC}=\mathrm{CPh}-\mathrm{CPh}=\mathrm{CPhC}_{6} \mathrm{H}_{4} \mathrm{CH}_{2} \mathrm{~N}\left(\mathrm{CH}_{3}\right)_{2}\right\}(\mathrm{NCO})\right](\mathbf{3})$ & 2220 vs & $1315 \mathrm{w}$ & - & $606 \mathrm{w}$ & $1595 \mathrm{~m}$ \\
\hline$\left[\mathrm{Pd}\left\{\mathrm{PhC}=\mathrm{CPh}-\mathrm{CPh}=\mathrm{CPhC}_{6} \mathrm{H}_{4} \mathrm{CH}_{2} \mathrm{~N}\left(\mathrm{CH}_{3}\right)_{2}\right\}\left(\mathrm{NO}_{3}\right)\right] \cdot 1,5 \mathrm{CH}_{2} \mathrm{Cl}_{2}(\mathbf{4})$ & $\ldots$ & - & 1394 vs, $1267 \mathrm{w}$ & - & $1590 \mathrm{~m}$ \\
\hline
\end{tabular}

MeCN: 2308 w, 2249 w, cm ${ }^{-1}$ 
do que o mesmo foi completamente deslocado da esfera de coordenação de paládio na reação com o difenilacetileno (dfa).

$\mathrm{O}$ espectro de RMN de ${ }^{13} \mathrm{C}$ do complexo (3) apresenta em $\delta=$ 151,21 um sinal correspondente ao carbono da ligação C(1)-Pd , em $\delta=87,02$ o sinal do carbono $\mathrm{C}(2)$ e entre $\delta=140,01$ a 125,65 sinais atribuídos aos carbonos aromáticos da dmba e dos grupos fenilas do acetileno. Em $\delta=66,91$ verifica-se o sinal referente ao carbono do grupo $\left(-\mathrm{N}_{-} \mathrm{CH}_{2}-\right)$ e em $\delta=51,71$ e 45,89 às metilas da dmba. Em $\delta=$ 111,07 observa-se um sinal atribuído aos carbonos C(3) e C(4) da dupla ligação, coordenados ao paládio, deslocados em relação ao sinal dos carbonos da tripla ligação, $\mathrm{C} \equiv \mathrm{C}$, do difenilacetileno livre em $\delta=123,26$. Este fato evidencia o efeito da perda do caráter de tripla ligação do dfa pela coordenação ao metal originando o produto de bis-inserção, bem como a interação da dupla ligação resultante com o átomo de paládio.

O espectro de RMN de ${ }^{1} \mathrm{H}$ mostra na região de $\delta=7,23$ a 6,53 os sinais atribuídos aos hidrogênios da dmba e dos grupos fenilas do acetileno. Em $\delta=2,66$ aparece um duplo dubleto correspondente ao grupo $\left(-\mathrm{N}-\mathrm{CH}_{2}-\right)$ e dois sinais em $\delta=2,55$ e 2,25 , indicativos da perda da equivalência dos grupos metila da dmba.

Considerando os resultados anteriores, é ilustrada na Figura 2 a estrutura do composto (3), resultante da bis-inserção de dfa na ligação Pd-C(5) de (1) .

$\mathrm{O}$ espectro de RMN de ${ }^{13} \mathrm{C}$ do composto (4) mostra em $\delta=148,6$ o sinal correspondente ao carbono da ligação C(1)-Pd, em $\delta=85,1$ o sinal do carbono $\mathrm{C}(2)$ e entre $\delta=146,4$ a 125,8 os sinais referentes aos carbonos aromáticos da dmba e dos grupos fenilas do acetileno de partida. Em $\delta=108,0$ nota-se um sinal dos carbonos C(3) e C(4) originários do difenilacetileno em $\delta=123,26$. Os sinais referentes ao ligante dmba não apresentam deslocamentos significativos em relação ao composto (3).

O espectro de RMN de ${ }^{1} \mathrm{H}$ mostra na região de $\delta=7,71$ a 6,57 os sinais atribuídos aos hidrogênios do dmba e dos grupos fenilas do acetileno. $\operatorname{Em} \delta=2,91$ e $\delta=2,71$ aparecem os sinais correspondentes ao grupo $\left(-\mathrm{N}_{-} \mathrm{CH}_{2}-\right)$ e dois sinais em $\delta=2,54$ e 2,35 indicativos da perda da equivalência dos grupos metila da dmba.

A Figura 3 representa a estrutura do complexo (4), resultante da

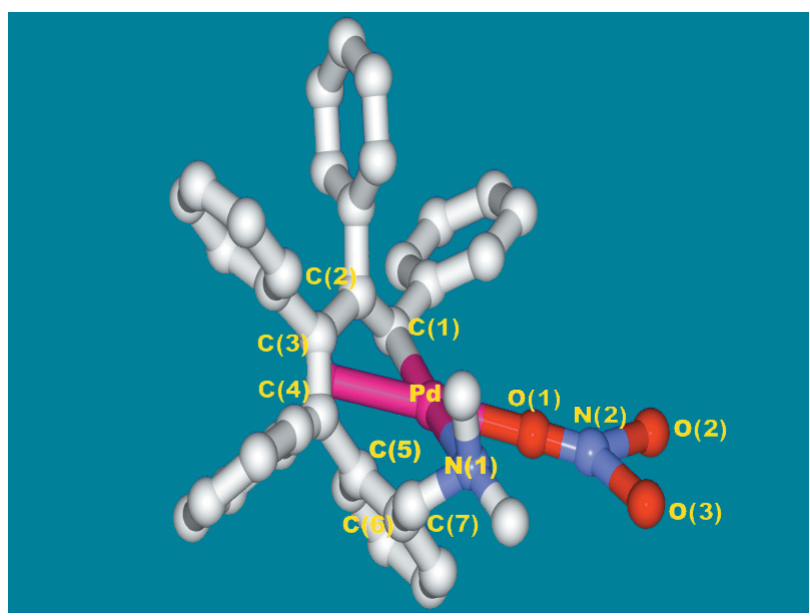

Figura 3. Produto de bis-inserção de difenilacetileno na ligação Pd-C no composto $\left[\mathrm{Pd}(\mathrm{dmba})(\mathrm{MeCN})_{2}\right]\left(\mathrm{NO}_{3}\right)$ reação de bis-inserção de difenilacetileno no ciclopaladado $\left[\mathrm{Pd}(\mathrm{dmba})(\mathrm{MeCN})_{2}\right]\left(\mathrm{NO}_{3}\right)$.

\section{CONCLUSÃO}

Neste trabalho foram realizadas, com sucesso, reações de inserção do 1,2-difenilacetileno na ligação Pd-C em ciclopaladados, um dimérico: o $[\mathrm{Pd}(\mathrm{dmba})(\mu-\mathrm{NCO})]_{2}(\mathbf{1})$ e o outro monomérico: o $\left[\mathrm{Pd}(\mathrm{dmba})(\mathrm{MeCN})_{2}\right]\left(\mathrm{NO}_{3}\right)(\mathbf{2})$.

A inserção de duas moléculas do alcino levou à formação de interessantes estruturas metalociclas (3) e (4), consistindo em anéis com nove átomos quelando o metal via ligações Pd-N(1) e Pd-C(1), estando os átomos $\mathrm{N}(1)$ e C(1) em posições trans. Há, também, um arranjo trans do grupo cianato e nitrato com relação à ligação Pdolefina, ou seja com relação à dupla ligação $\mathrm{C}(3)=\mathrm{C}(4)$ do produto de inserção. Cabe salientar que estas reações de bis-inserção constituem um método eficiente para a síntese de novos compostos orgânicos que não podem ser obtidos por métodos convencionais.

\section{AGRADECIMENTOS}

Os autores agradecem ao CNPq, CAPES e FAPESP pelo suporte financeiro.

\section{REFERÊNCIAS}

1. Yatsimirsk, A. K.; Kanzakov, G. M.; Ryabov, A. D.; J. Chem. Soc., Perkin Trans. 2 1992, 8, 1295; Camargo, M.; Dani, P.; Dupont, J.; De Souza, R. F.; Pfeffer, M.; Tkatchenko, I.; J. Mol. Catal. A:Chem. 1996, 109, 127; Herrmann, W. A.; Böhm, V. P. W.; Reinsinger, C. P.; J. Organomet. Chem. 1999, 576, 23.

2. Ghedini, M.; Pucci, D.; Cesarotti, E.; Antonigzza, P.; Francescangeli, O.; Bartolino, R.; Chem. Mat. 1993, 5, 883; Espinet, P.; Esteruelas, M. A.; Oro, L. A.; Serrano, J. L.; Sola, E.; Coord. Chem. Rev. 1995, 117, 215; Saccomando, D. J.; Black, C.; Cave, G. W. V.; Lydon, D. P. ; Rourke, J. P.; J. Organomet. Chem. 2000, 601, 305.

3. Higgius, J. D.; J. Inorg. Biochem. 1993, 49,149; Navarro-Ranninger, C.; López-Solera, I.; González, V. M.; Pérez, J. M.; Alvarez-Valéz, A.; Martin, A.; Raibithby, P. R. ; Masaguer, J. R., Alonso, C.; Inorg. Chem. 1996, 35, 5181; Caires, A. C. F.; De Almeida, E. T.; Mauro, A. E.; Hemerly, J. P.; Valentini, S. R.; Quim. Nova 1999, 22, 329.

4. Chorodowski-Kimmes, S.; Beley, M.; Collin, J. P.; Sauvage, J. P.; Tetrahedron Lett. 1996, 37, 2963; Carina, R. F.; Willians, A. F.; Bernardinelli, G.; J. Organomet. Chem. 1997, 548, 45.

5. Trofimenko, S.; Inorg. Chem. 1973, 12, 1215.

6. Ryabov, A. D. Em Perspectives in Coordination Chemistry; Willians, A. F.; Floriani, C.; Merbach, A. E., eds.; Verlag Helvetica-Chimica Acta: Basel, 1992.

7. Ryabov, A. D.; Sakodinskaya, I. K.; Yatsimirsky, A. K.; J. Chem. Soc., Perkin Trans. 2 1983,10, 1518

8. Câmpora, J.; Palma, P.; Carmona, E.; Coord. Chem. Rev. 1999,193-195, 207.

9. Ryabov, A. D.; Synthesis 1985, 3, 233.

10. Pfeffer, M.; Dupont, J.; J. Organomet. Chem. 1987, 321, 13.

11. Mauro, A. E.; Caires, A. C. F.; Santos, R. H. A.; Gambardella, M. T. P.; J. Coord. Chem. 1999, 48, 521.

12. Santana, A. M.; Mauro, A. E.; De Almeida, E. T.; De Godoy Netto, A.V.; Klein, S. I.; Santos, R. H. A.; Zóia, J. R.; J. Coord. Chem. 2001, 53, 163.

13. Lucca Neto, V. A.; Mauro, A. E.; Caires, A. C. F.; Ananias, S. R.; De Almeida, E. T.; Polyhedron 1998, 18, 413.

14. Ananias, S. R.; Mauro, A. E.; Nogueira, V. M.; Haddad, P. S.; De Almeida, E. T.; Eclética Química 2002, 26, 86.

15. Nakamoto, K.; Infrared and Raman Spectroscopy of Inorganic and Coordination Compounds, $4^{\text {th }}$ ed., Wiley Interscience: New York, 1986. 